\title{
ADVERSARIAL RISK ANALYSIS FOR FIRST-PRICE SEALED-BID AUCTIONS
}

\author{
MUHAMMAD EJAZ $\mathbb{D}$
}

(Received 23 December 2021; first published online 8 February 2022)

\begin{abstract}
2020 Mathematics subject classification: primary 62C12; secondary 91A35.
\end{abstract}
Keywords and phrases: adversarial risk analysis, game theory decision theory.

First-price sealed-bid (FPSB) auctions have mostly been modelled in auction theory using the decision-theoretic and Bayesian game-theoretic approaches. From a practical point of view, each approach has its limitations. To overcome those limitations, [4] introduced an approach, called adversarial risk analysis (ARA) that provides an optimal solution for one of the intervening agents, based on a decision making problem in hand and treating the intelligent adversaries' decisions as uncertainties. ARA solutions for FPSB auctions have previously been found but only under strong assumptions, which make the model somewhat unrealistic.

In this thesis, we use ARA methodology and model bidders' behaviours in FPSB auctions using more realistic assumptions. First, we model bidders' behaviours by defining a new utility function that considers bidders' wealth, which is assumed to be different for each bidder. We consider bidders' wealth since it is a significant determinant of their bidding behaviour in these auctions. Also, we define new risk behaviour parameters that change with the relative change in circumstances of bidders' wealth. In our modelling, we assume that the auctioned item is normal and has a reserve price which is known in advance to each bidder. We find ARA solutions not only for risk-neutral but also for risk-averse as well as risk-seeking bidders. We model these auctions by ARA framework using nonstrategic play, level- $k$ thinking, mirror equilibrium (ME) and Bayes-Nash equilibrium (BNE) solution concepts. For finding ARA solutions using nonstrategic play and level- $k$ thinking, ME and BNE (asymmetric case) solution concepts, we assume two bidders. Whereas we assume $n$ bidders while finding ARA solutions using the BNE symmetric case.

Second, we use ARA methodology and model bidders' behaviours using the utility function that takes into account bidders' winning and losing regret for the auctioned

Thesis submitted to the University of Waikato in April 2021; degree approved on 12 August 2021; supervisors Chaitanya Joshi and Stephen Joe.

(C) The Author(s), 2022. Published by Cambridge University Press on behalf of Australian Mathematical Publishing Association Inc. 
item. We define new winning and losing regret parameters and a modified utility function to take into account the effect of bidders' wealth on their bidding behaviours. Using the modified utility function, we find ARA solutions using nonstrategic play and level- $k$ thinking solution concepts assuming $n$ bidders participating in these auctions.

We give numerical examples to illustrate our methodology.

Some of this research has been published in [1-3].

\section{References}

[1] M. Ejaz, S. Joe and C. Joshi, 'Adversarial risk analysis for auctions using mirror equilibrium and Bayes Nash equilibrium', Decis. Anal. 18(3) (2021), 185-202.

[2] M. Ejaz, C. Joshi and S. Joe, 'Adversarial risk analysis for first-price sealed-bid auctions', Aust. N. Z. J. Stat. 63(2) (2021), 357-376.

[3] M. Ejaz, C. Joshi and S. Joe, 'Adversarial risk analysis for auctions using non-strategic play and level- $k$ thinking: a general case of $n$ bidders with regret', Comm. Statist. Theory Methods, to appear.

[4] D. Ríos Insua, J. Rios and D. Banks, 'Adversarial risk analysis', J. Amer. Statist. Assoc. 104(486) (2009), 841-854.

MUHAMMAD EJAZ, Department of Mathematics, University of Waikato, Hamilton 3216, New Zealand

e-mail: me64@students.waikato.ac.nz 